\title{
Virologic Null-Response
}

National Cancer Institute

\section{Source}

National Cancer Institute. Virologic Null-Response. NCI Thesaurus. Code C120604.

The subject remains viremic during the treatment period and the decrease in viral load from baseline at a specified timepoint is below or equal to a predetermined threshold. 